\title{
Privato, pubblico e aperto
}

Citation:S. Penge(2021) Privato, pubblico e aperto. Media Education 12(2): 15-24. doi: $10.36253 / \mathrm{me}-11442$

Received: June, 2021

Accepted: October, 2021

Published: December, 2021

Copyright: $\odot 2021$ S. Penge. This is an open access, peer-reviewed article published by Firenze University Press (http://www.fupress.com/me) and distributed under the terms of the Creative Commons Attribution License, which permits unrestricted use, distribution, and reproduction in any medium, provided the original author and source are credited.

Data Availability Statement: All relevant data are within the paper and its Supporting Information files.

Competing Interests: The Author(s) declare(s) no conflict of interest.

\section{Private, public and open}

\author{
Stefano Penge \\ Lynx Srl \\ stefano.penge@gmail.com
}

\begin{abstract}
In this text we try to clarify some misunderstandings that in our opinion have arisen on the issue of digital educational platforms. These misunderstandings are based on the one hand on the profound misconception of the meaning of "open", which is reduced to "free", and on the other hand on a conception of the company as an activity necessarily extraneous to the ethical dimension. Overcoming these misunderstandings could lead to a collaboration between private and public, between profit and non-profit that defines precisely the models and standards and lays the foundations for the construction of an ecosystem of open, inter-operating and ready-to-use platforms both on the software side and on the content side.
\end{abstract}

Keywords: ecosystem, ethics, free, open, platforms.

Riassunto. In questo testo proviamo a chiarire alcuni equivoci che a nostro parere si sono generati a proposito della questione delle piattaforme digitali educative. Si tratta di equivoci che poggiano da un lato sull'incomprensione profonda del significato di "aperto", che viene ridotto a "gratuito", e dall'altro su una concezione dell'impresa come attività necessariamente estranea alla dimensione etica. Superando questi equivoci si potrebbe giungere ad una collaborazione tra privato e pubblico, tra profit e no-profit che definisca in maniera precisa i modelli e gli standard e getti le basi per la costruzione di un ecosistema di piattaforme aperte, inter-operanti e predisposte per il riuso e la personalizzazione sia dal lato software che da quello dei contenuti.

Parole chiave: ecosistema, etica, free, open, piattaforme.

\section{PUBBLICO CONTRO PRIVATO}

"Public education cannot be dependent on digital platforms provided by private companies" (ICFE 2020, p. 17).

Questa dichiarazione è tratta dal rapporto della Commissione Internazionale dell'UNESCO per il Futuro dell'Educazione "Education in a postCOVID world: Nine ideas for public action”. Il documento pone l'attenzione 
sull'importanza di agire in maniera globale nel settore dell'educazione per contrastare le ingiustizie che sono state amplificate dalla pandemia. Lo fa in nove punti, uno dei quali, il sesto, è dedicato a riportare il centro della riflessione e dell'azione sul rapporto umano tra docenti e studenti, a contrastare il predominio della tecnologia digitale e la proposizione di contenuti "ready made" e a proporre una modalità aperta di costruzione e distribuzione di risorse educative.

Mentre gli obiettivi del documento sono largamente condivisibili, è proprio sull'idea numero 6 che vogliamo concentrarci, per evidenziare quelli che a nostro avviso possono essere degli equivoci che rischiano di nuocere alle stesse finalità di fondo del documento.

La frase citata in apertura rappresenta una presa di posizione forte: l'uso di cannot, al posto di un più permissivo should not, traccia un muro tra i servizi educativi erogati dagli enti pubblici e i servizi digitali (le platforms, qualunque cosa voglia dire questo termine) erogati da imprese private. La dichiarazione definisce una doppia contrapposizione: tra pubblico e privato, da un lato, e tra educazione e digitale dall'altro. In generale, le imprese del comparto digitale vengono considerate in opposizione ai soggetti pubblici, come esterne al pedagogical space (ICFE, p. 8). Non è previsto che un'impresa a fini di lucro possa essere interessata a lavorare al bene comune in campo educativo, o che possa rinunciare ad un maggiore profitto in nome di valori etici.

Nel paragrafo riassuntivo dell'idea 6 la Commissione richiede una "global collaboration among governments, philanthropy, and non-profit organizations to develop and distribute open educational resources and open platforms". C'è un fine da raggiungere (lo sviluppo e la distribuzione di OER e di piattaforme aperte) e un mezzo (la collaborazione tra tutti gli enti, meno quelli profit). Ancora più nettamente, le imprese vanno addirittura estromesse dal settore dell'educazione pubblica: "[...] much of what is currently provided by private companies should become a public undertaking [...]" (ICFE 2020, p. 16).

Che cosa si intenda per "open" è spiegato poco dopo: "Here, our efforts should focus on open licensing and open access policies that facilitate no-cost use, reuse, repurposing and adaption" (ICFE 2020, p. 17).

Ecco il motivo per cui le imprese sono escluse dalla chiamata a collaborare: perché lo scopo dei soggetti for profit non può essere quello di sviluppare e distribuire risorse e piattaforme aperte. Le imprese, nella visione sottesa al documento, sono solo quelle che adottano il modello di business dell'offerta di servizi a pagamento. In particolare, le imprese digitali sono centrate sulle tecnologie e non sul miglioramento della vita delle persone ("advancing the interests and capabilities of learners"). Le imprese digitali sono esterne allo spazio pedagogi$\mathrm{co}$, ma forse si può dire che la stessa tecnologia digitale è esterna alla pedagogia: è un mezzo, alla pari di tanti altri che l'hanno preceduto, come la stampa e la televisione. Questa posizione nei confronti del digitale - che si potrebbe chiamare di neutralità tecnologica - è molto diffusa nell'ambiente educativo, in particolare tra i docenti meno favorevoli al suo utilizzo. Il digitale viene pensato come un medium indifferente, di per sé vuoto, una corrente che trasporta contenuti, come l'elettricità: a questi patti può essere utilizzato anche da chi non lo apprezza. La mente va naturalmente al motto di McLuhan: difficile immaginare che il medium non modifichi il messaggio.

"We must ensure that any digital transition is not just an effort pushed by technology companies but that teachers, students, governments, civil society representatives and privacy advocates are also represented and shape these transformations" (ICFE 2020, p. 17).

Sembra di leggere tra le righe un modello di impresa digitale che abbiamo tutti ben presente: un'impresa di dimensioni planetarie, con un fatturato superiore al bilancio di tanti Stati, che fornisce servizi in tutti i settori, che incrocia i dati da tutti questi servizi e si pone come obbiettivo quello di controllare la vita di ogni persona sul pianeta. Un vero Leviatano a cui tutti abbiamo conferito potere un po' alla volta, volontariamente.

Ma questo non è l'unico modello possibile di impresa digitale che lavora nel campo dell'educazione. Esiste una lunga storia di imprese che hanno lavorato nella ricerca, nella progettazione e nello sviluppo di software educativo, di ambienti digitali, di linguaggi, di contenuti didattici. Molte hanno partecipato alla nascita delle applicazioni del digitale all'educazione allo stesso titolo dei centri di ricerca e delle università. Lavorando al confine tra ricerca e sviluppo, queste imprese hanno intrecciato pedagogia e informatica in maniera più stretta di quanto non immaginino i docenti di oggi. La storia dei linguaggi di programmazione educativi, ad esempio, è ricca di esempi di ambienti che non sono semplicemente idee pedagogiche concretizzate in forma digitale, ma sono anche idee digitali orientate pedagogicamente. Si pensi al linguaggio Logo, precursore di tanti ambienti dedicati al coding come Scratch e Snap!, realizzato alla Bolt Beranek and Newman di Cambridge nel 1967. Logo non avrebbe potuto essere così innovativo nel proporre un modello d'uso in cui il bambino insegna nuove parole al computer se non fosse stato pensato a partire da un linguaggio funzionale (il Lisp), in cui le funzioni sono oggetti di prima classe, ovvero sono manipolabili e costruibili dentro ad un programma. Questi ambien- 
ti felici hanno prodotto grandi risultati perché hanno saputo incrociare saperi pedagogici e digitali mettendo insieme team bilanciati di ricercatori ed esperti in entrambi i campi: Feurzeig, Solomon, Papert.

Molte di queste società sono scomparse o si sono trasformate, poche sono rimaste in vita. Alcune sono passate nel campo dell'open source e hanno iniziato a rilasciare i propri prodotti con una licenza aperta. Ma che significa "licenza aperta"?

\section{LA PAROLA "OPEN"}

La parola open sta forse andando un po' troppo oltre il suo significato originario. In un articolo del 2013 di Evgenij Morozov (che peraltro fa parte della Commissione UNESCO) questa tendenza ad usare "aperto" solo come una connotazione positiva, quasi priva di significato, viene stigmatizzata come una moda, anzi una religione, esattamente come il "green":

"Openness is today a powerful cult, a religion with its own dogmas. [...] This fascination with "openness" stems mostly from the success of open-source software, publicly accessible computer code that anyone is welcome to improve. But lately it has been applied to everything from politics to philanthropy. For many institutions, "open" has become the new "green." (Morozov, 2020).

Morozov prova a distinguere significati diversi di "open", e riconosce che il termine deve il suo successo alla vicenda dell'open source, ma viene usato a proposito della società (Popper), della politica (Osborne) fino alle bevande gassate. Avvicinandosi al nostro tema, Morozov sostiene che anche i MOOC sarebbero davvero open se permettessero il remix dei contenuti, cosa che non è sempre possibile.

Ma se volessimo ripartire dall'origine, dovremmo confrontare "free software" con "open source": espressioni nate in momenti diversi - la prima venti anni prima della seconda - e in contesti culturali diversi, che fanno riferimento a modelli diversi. Mentre il free software nasce come richiesta etica in reazione ad un'azione economica (l'imposizione di una licenza proprietaria ad un codice sorgente nato come libero), l'open source è una risposta tecnica ad un problema tecnico: se il codice sorgente è leggibile, il software che ne risulta è qualitativamente migliore perché è frutto del lavoro di tanti.

Il concetto di software libero nasce da un rovesciamento del diritto d'autore in un permesso d'autore (il termine originario è "copyleft" ed è in uso dai primi anni '70): l'autore concede a chi entra in possesso del codice sorgente alcuni diritti, ovvero quelli di esecuzio- ne, lettura, modifica, distribuzione. La definizione canonica $^{1}$ dei permessi - le libertà - che l'autore di un software concede a chi ne entra in possesso richiama alla mente di ogni americano le quattro libertà di Roosevelt (libertà di espressione, libertà religiosa, libertà dal bisogno e libertà dalla paura). Anche nel caso del software, le prime due libertà riguardano l'individuo e le seconde due il rapporto con la comunità (Free Software Foundation, 2021):

1. Libertà di eseguire il programma come si desidera, per qualsiasi scopo.

2. Libertà di studiare come funziona il programma e di modificarlo in modo da adattarlo alle proprie necessità.

3. Libertà di ridistribuire copie in modo da aiutare gli altri.

4. Libertà di migliorare il programma e distribuirne pubblicamente i miglioramenti da voi apportati (e le vostre versioni modificate in genere), in modo tale che tutta la comunità ne tragga beneficio.

Le libertà 1 e 3 , in particolare, richiedono l'accesso al codice sorgente, cioè la possibilità di leggerlo. In pratica, tutti i software che adottano una licenza free software sono anche open source. Ma si capisce come il modello del software libero vada al di là della richiesta di leggibilità del codice, che invece è l'unica veicolata dall'espressione "a sorgente aperta"2.

\section{GRATUITÀ E SOSTENIBILITÀ}

Un'equazione diffusa vuole che il software libero debba necessariamente essere gratuito, cioè che non possa essere venduto. E infatti molti software usati e conosciuti da tutti sono rilasciati con una licenza libera, come la Gnu Public License (GPL), e sono scaricabili gratuitamente, rendendo possibile a milioni di persone di inviare email, scrivere documenti, navigare nel web senza pagare per l'acquisto di una licenza. In questi casi è difficile che ci si fermi a domandarsi: come è possibile? Come può esserci un artefatto, un prodotto dell'ingegno umano che, pur avendo richiesto lavoro per essere costruito, non ha bisogno di ripagare questo lavoro? Certo, si può immaginare che ci siano schiere di volontari che dedicano parte del loro tempo libero a scrivere software come passatempo, o per vantarsene con gli amici. Ma è pensabile che

\footnotetext{
${ }^{1}$ https://www.gnu.org/philosophy/free-sw.html

${ }^{2} \mathrm{Nel}$ seguito di questo articolo faremo riferimento solo al termine open source, che è quello usato nel documento da cui siamo partiti, e anche nella maggior parte delle conversazioni, e useremo l'acronimo F/LOSS (Free/Libre Open Source Software) per indicare collettivamente tutto il software libero e aperto.
} 
questo modello abbia permesso di realizzare Gnu Linux, o Apache, o tanti altri software open source, senza i quali oltre la metà dei siti web sarebbe spenta?

Ci sono due premesse interessanti sotto questa equazione. La prima è quella che riconosce al software la natura di oggetto di conoscenza, non di puro strumento. Il software è un bene immateriale, esattamente come la letteratura o la mappa del codice genetico di un organismo naturale. La seconda premessa è quella che sottolinea l'aspetto etico della condivisione: il software (libero) deve essere un bene universale, di tutti, perché tutti devono avere gli stessi diritti di godere di quel bene. Da queste due premesse deriverebbe la gratuità necessaria del software libero.

Curiosamente, queste due premesse non sono sempre collegate. Ad esempio, la fruizione di un'opera letteraria (che è un bene immateriale) può essere gratuita per un lettore solo perché qualcuno - una biblioteca - ha pagato un prezzo per l'acquisto di una copia, perché si sa che la sua produzione, la riproduzione e la distribuzione hanno dei costi. Si discute, nel caso della forma elettronica dell'ebook, che elimina quasi del tutto i costi relativi al supporto, se la distribuzione dei ricavi tra autore, editore e distributore sia davvero proporzionale ai costi; ma non si mette in discussione il principio di fondo, né si richiederebbe agli autori di lavorare gratis.

Probabilmente questo deriva dal fatto che mentre abbiamo una qualche idea del fatto che scrivere un libro (o una canzone, o un film) sia un lavoro lungo e faticoso, che richiede competenze che si apprendono nel tempo, scrivere il software è invece un'attività di cui abbiamo al massimo sentito parlare, e a cui non riconosciamo lo stesso valore intellettuale. Inoltre, di solito abbiamo un'idea del processo produttivo che parte dalle dita dell'autore e termina nelle pagine del libro; mentre non abbiamo nessuna idea di come nasce un programma e del processo per il quale diventa quella cosa che fa girare il mondo.

Per sommi capi: normalmente, per creare un programma si scrive il suo codice, riga dopo riga, in uno degli ottomila linguaggi di programmazione esistenti. Questa operazione dura giorni, mesi, a volte anni; ed è spesso opera di più persone contemporaneamente, o in successione. Alla scrittura seguono di solito delle fasi ulteriori di collegamento di funzioni standard, di verifica della correttezza sintattica e semantica, di traduzione in formati intermedi, fino alla compilazione, cioè alla trasformazione del codice in un oggetto eseguibile direttamente e velocemente, senza bisogno di altro supporto, su una macchina diversa da quella su cui è stato scritto.

Il prodotto finale (il programma eseguibile) non è più scritto nel linguaggio di programmazione originario, ma in un linguaggio composto di istruzioni semplicis- sime eseguibili dal processore, e di solito non permette di risalire al codice sorgente. Per questo motivo, la compilazione è anche una forma di assicurazione sulla proprietà del codice stesso da parte dell'autore. Nel caso del software proprietario (cioè non libero) la procedura inversa, cioè la decompilazione, è vietata esplicitamente dal contratto con il quale il software è venduto. Nel caso del software libero, invece, l'autore non si preoccupa della possibilità che altri possano leggere il suo codice, o anche modificarlo. Anzi: può solo augurarsi che questo avvenga, perché così il suo lavoro riceve dei miglioramenti che da solo non avrebbe potuto apportare. Naturalmente però vorrebbe essere pagato per continuare il suo lavoro: ma non nella forma della vendita della licenza d'uso, bensì nella forma di contratto di consulenza, installazione, personalizzazione, formazione, intorno al suo programma. Sono i servizi al contorno che vengono pagati, non l'oggetto in sé.

Che la scelta del termine "free", che in inglese può significare tanto "libero" che "gratis", avrebbe dato origine a fraintendimenti lo sapeva bene Richard Stallmann, che specificava "Free as free speech, not as free beer". Ma sapeva anche che impedire di vendere il software libero sarebbe stato da un lato una sottrazione di libertà, e dall'altro un ostacolo enorme per la sua diffusione:

"Software libero" non vuol dire non commerciale. Al contrario, con un programma libero deve essere possibile anche l'uso commerciale, lo sviluppo commerciale, e la distribuzione commerciale. Questa politica è di importanza fondamentale - senza questa il software libero non potrebbe raggiungere i suoi obiettivi." (Free Software Foundation, 2021)

Nel testo della Commissione UNESCO da cui siamo partiti, però, free e open source sembrano essere come sinonimi. E in effetti nella formulazione sintetica dell'idea numero 6 vengono usati insieme, senza distinzioni: "Make free and open source technologies available to teachers and students". Sembrerebbe che il significato sottostante, comune ad entrambi, sia quello di "liberamente accessibile", cioè di gratuito. Questo si ricava dalla frase immediatamente seguente, che unisce tutte le risorse educative in un'unica richiesta: "Open educational resources and open access digital tools must be supported". Non open digital tools, ma open access digital tools.

Che significa supportare uno strumento? Finanziarne lo sviluppo, e la manutenzione, oppure finanziare pubblicamente i servizi basati su quello strumento? O semplicemente sostenere che non dovrebbero essere sostenuti costi per il suo utilizzo? Sono chiaramente scelte molto diverse, che possono portare alla scompar- 
sa dello strumento in questione per l'esaurimento delle risorse economiche necessarie al suo mantenimento.

Se la richiesta di rendere gratuite tutte le risorse educative, comprese le piattaforme digitali, ci lascia francamente interdetti, sarebbe invece del tutto condivisibile l'affermazione secondo la quale le piattaforme digitali da cui dipende l'educazione pubblica dovrebbero essere aperte, nel senso preciso che il software con cui vengono realizzati i servizi digitali che consentono quelli educativi deve avere una licenza F/LOSS. Perché? Perché in questo modo si avrebbe la garanzia che il codice sorgente sia leggibile, cioè controllabile per verificare che non ci siano parti nascoste che effettuano operazioni illecite, come la raccolta di dati personali e la loro rivendita; integrabile con altri codici sorgenti - purché rilasciati con licenze compatibili - ma anche modificabile, cioè adattabile a contesti e utenti diversi, e migliorabile, in funzione di nuove esigenze che sicuramente emergeranno. Ci si assicurerebbe che l'investimento fatto all'inizio non venga perso e che non ci sia nessun lock-in (quel fenomeno per il quale una volta adottata una tecnologia non è possibile passare ad altro fornitore), perché chiunque ne sia capace tecnicamente può modificare e migliorare il software.

Tutte queste caratteristiche dovrebbero caratterizzare le piattaforme digitali, e in generale tutti i software che vogliano proporsi nell'educazione pubblica; ma non c'è una correlazione necessaria con la richiesta che i servizi basati su questi software debbano essere anche gratuiti. Se un soggetto (un'azienda, una cooperativa, un consorzio, pubblico o privato) fornisce servizi che implicano dei costi - hosting, banda, manutenzione, assistenza; ma anche personalizzazione e formazione - è normale che questi costi siano riversati sui clienti. Anzi: l'esistenza di un contratto e di uno scambio tra servizio e denaro è (entro certi termini) una modalità per assicurare entrambi i soggetti. Di fatto, uno degli ostacoli all'adozione del software libero da parte dei soggetti pubblici è proprio l'assenza di un fornitore che, all'interno di un contratto di servizio, garantisca assistenza e manutenzione di un prodotto software. Dall'altro lato, un contratto è un modo per ripagare il fornitore del tempo speso anche solo per selezionare uno strumento open source, per studiarlo e personalizzarlo, oltre che per fornire servizi basati su quello.

Quali potrebbero essere le alternative? Come sarebbe sostenibile un'offerta gratuita di servizi digitali per l'educazione pubblica? Vediamo due esempi dal panorama italiano.

\subsection{Servizi digitali offerti da un ente pubblico}

E quanto propone il disegno di legge della senatrice Laura Mantovani “Istituzione della Rete di interconnes- sione unica nazionale dell'istruzione - UNIRE", seguita da altri, che indica in particolare nel GARR il soggetto a cui dovrebbe essere data in gestione la fornitura di servizi, non solo puramente tecnici (la banda, i DNS) ma anche educativi. L'intento del DDL è quello di creare un cloud privato di proprietà dalla PA italiana per sostenere la digitalizzazione dei servizi della scuola, ma anche di sviluppare e fornire il servizio unico nazionale per la didattica digitale integrata.

Tuttavia, una piattaforma per la didattica non è un'infrastruttura neutra. C'è una differenza enorme tra i servizi amministrativi, o quelli informatici come i DNS, e quelli didattici. I servizi didattici si rivolgono a studenti, che sono persone, non enti o macchine. Quindi devono essere pensati per loro e intorno a loro, in termini di interfaccia, di linguaggio, di funzionalità (che è proprio ciò che chiede il Documento della Commissione UNE$\mathrm{SCO}$ ). Una piattaforma per la didattica dovrebbe essere adatta a persone diverse e a contesti diversi (corsi obbligatori e facoltativi, corsi teorici e pratici, laboratori e corsi che si costruiscono dal basso). Insomma, un oggetto molto complesso - e quindi costoso - da progettare, da realizzare; ma soprattutto da gestire, da manutenere, da migliorare, da integrare con nuove tecnologie ancora nemmeno all'orizzonte. Quanto potrebbe durare, una volta esaurito il finanziamento iniziale, un sistema di questo genere? Quanto impiegherebbe a diventare obsoleto e inutilizzabile?

E vero che alcuni Paesi europei si sono mossi proprio in questa direzione, come è il caso della Francia ${ }^{3}$, ma anche di Portogallo ${ }^{4}$ e Irlanda ${ }^{5}$. Si tratta però spesso di raccolte di risorse, o di MOOC, più che di vere piattaforme didattiche che permettono un'interazione tra studenti e docenti di una classe.

\subsection{Servizi gratuiti offerti da imprese private}

E quello che veniva richiesto dal MIUR nella sua pagina dedicata alle risorse per affrontare l'emergenza COVID $-19 .^{6}$

"I collegamenti delle varie sezioni di questa pagina consentono di raggiungere ed utilizzare a titolo totalmente gratuito le piattaforme e gli strumenti messi a disposizione delle istituzioni scolastiche grazie a specifici Protocolli siglati dal Ministero. Tutti coloro che vogliono supportare

\footnotetext{
${ }^{3}$ https://www.cned.fr/decouvrir-le-cned/espace-presse/ma-classe-a-lamaison

${ }^{4}$ https://apoioescolas.dge.mec.pt/

${ }^{5}$ https://www.pdst.ie/distancelearning

${ }^{6}$ Alla data in cui scriviamo, la pagina https://www.istruzione.it/coronavirus/didattica-a-distanza.html non è più raggiungibile.
} 
le scuole possono farlo aderendo alle due call pubblicate dal Ministero che contengono anche i parametri tecnici necessari." (MIUR, 2020)

I requisiti "tecnici" che devono avere le piattaforme sono in realtà un misto di varie cose, ma quello principale è che il servizio deve essere fornito gratuitamente garantendo però assistenza a docenti e studenti: "[...] tutte le piattaforme devono essere rese disponibili gratuitamente nell'uso e nel tutorial; la gratuità va intesa sia nella fase di adesione ed utilizzo dello strumento sia al termine di tale fase. Nessun onere, pertanto, potrà gravare sulle Istituzioni scolastiche e sull'Amministrazione" (MIUR, 2020).

Seguono dei requisiti diversi, a seconda dell'appartenenza della piattaforma all'una o all'altra di queste due macro-categorie:

1. "per le piattaforme di fruizione di contenuti didattici e assistenza alla community scolastica: sicurezza, affidabilità, scalabilità e conformità alle norme sulla protezione dei dati personali, nonché divieto di utilizzo a fini commerciali e/o promozionali di dati, documenti e materiali di cui gli operatori di mercato entrano in possesso per l'espletamento del servizio;

2. per le piattaforme di collaborazione on line: qualifica di "cloud service provider della PA" inerente alla piattaforma offerta, ai sensi delle circolari Agid n. 2 e 3 del 9 aprile 2018".

Sembra di capire che le piattaforme per la collaborazione online non siano soggette al divieto di utilizzo a fini commerciali e/o promozionali di dati. Siccome il riuso a fini commerciali o promozionali di dati è proprio una delle maniere con cui un fornitore di servizi digitali copre i propri costi e quindi rende sostenibile un servizio gratuito per l'utente finale, la domanda è allora in quale categoria vadano collocate piattaforme che sono costruite intorno a suite di strumenti per la produzione e condivisione di documenti, come Google Classroom e Microsoft Teams.

$\mathrm{Al}$ di là di questa suddivisione, sembra però evidente che solo dei soggetti veramente grandi possano permettersi di fare un'offerta gratuita che comprende l'assistenza a tutte le scuole italiane, cioè - ricordiamolo - a otto milioni di studenti, divisi in trecento settantamila classi. Perciò non stupiva di trovare ai primi tre posti nell'elenco delle piattaforme "raccomandate" dal MIUR Google Suite, MS Teams e TIM Weschool.

Un requisito che brilla per la sua assenza è invece quello del codice sorgente aperto. Nel contesto italiano, però, il requisito di apertura del codice non è semplicemente una raccomandazione legata al quadro generale, come nel Documento della Commissione UNESCO. In Italia la legge del 7 agosto 2012 n. 134 ha modificato l'art. 68 del Codice dell'Amministrazione Digitale introducendo per tutta la PA l'obbligo di effettuare "analisi comparativa di soluzioni", comprese quelle basata su software libero o codice sorgente aperto. Inoltre, nelle Linee Guida per l'adozione e il riuso del software da parte delle PA che sono in vigore dal 9 maggio 2019, si aggiunge, tra i criteri di valutazione, l'uso di dati aperti, di interfacce aperte e di standard per l'interoperabilità.

In conclusione, questo secondo modello, che sembra così attraente nell'immediato perché permette a istituzioni educative pubbliche di accedere a servizi digitali in maniera gratuita, mostra però, a nostro parere, i suoi limiti nel medio termine. Con questo genere di requisiti si esclude, di fatto, ogni piccolo fornitore locale, che sia una società tradizionale o una cooperativa di ex-studenti di un Istituto Tecnico. In questo modo viene favorito un modello monopolistico, in cui soggetti in grado di assorbire costi enormi sotto la voce di investimenti in comunicazione e fidelizzazione (nella migliore delle ipotesi) vedono scomparire tutti gli altri competitor piccoli e medi, fino a ritrovarsi come unica possibile offerta sul mercato. Si crea così un monopolio non solo di servizi, ma anche di linguaggio, di concetti, di modelli d'uso: il fatto che siano stati riproposti nel campo educativo, più o meno adattati, strumenti pensati per l'ufficio, sta anche cambiando il lessico ("meeting" anziché "lezione") e la maniera di pensare l'interazione didattica, che sempre di più si schiaccia nell'assegnazione di compiti e nella consegna di documenti di tipi predefiniti, realizzati con strumenti digitali progettati per adulti e per il loro lavoro d'ufficio, come un word processor, un foglio di calcolo o un software per realizzare lucidi.

L'altro effetto negativo di questa seconda modalità è la progressiva perdita di competitività del comparto digitale in Italia. Sono sempre meno i settori in cui abbia senso investire idee e lavoro, e l'ultimo settore è proprio quello dell'educazione, dove non ha senso immaginare di competere con i giganti che sono scesi in campo proprio in occasione della pandemia. Le imprese digitali, anche quelle del settore open source, finiscono per dedicarsi all'importazione di software e alla costruzione di soluzioni basate su quelli, più che al progetto e allo sviluppo di software originale. Non c'è bisogno di essere ferventi nazionalisti per considerare questa mancanza di prospettive un grosso ostacolo alla diffusione di una cultura dello sviluppo di software tra i giovani.

\section{RISORSE APERTE}

L'altro obiettivo polemico del punto 6 del Documenti ICFE è quello del supermercato delle risorse pronte per l'uso, in vendita sugli scaffali virtuali: "Education 
cannot thrive with ready-made content built outside of the pedagogical space and outside of human relationships between teachers and students" (ICFE 2020, p. 6). Al loro posto si propone di usare "digital tools in teachers' pedagogical work with students" partendo però da risorse gratuite, per le quali siano possibili "reuse, repurposing and adaption". Andrebbe analizzato cosa si intende per digital tools, perché si tratta comunque di software, e anche questi software devono essere progettati, sviluppati e mantenuti da qualcuno. Mentre le piattaforme oggi dominano il discorso sull'educazione con il digitale, i software educativi sono un po' scomparsi all'orizzonte, sostituiti da app generiche, gratuitamente accessibili su web, su cui però valgono gli stessi discorsi a cui abbiamo accennato sopra: qual è il loro modello di business? Come si sostengono? Come garantiscono la privacy degli utenti? Ci piacerebbe che il documento della Commissione dichiarasse, in linea con i suoi obiettivi generali, che anche questi digital tools non devono essere strumenti generici nati per altre finalità, ma devono essere ambienti progettati appositamente per l'apprendimento, insieme a docenti e studenti, in uno spazio pedagogico.

Il discorso sugli oggetti d'apprendimento è abbastanza datato, e rimanda alla discussione su un vecchio standard dell'ADL: lo SCORM (Shared Content Object Reference Model) $)^{7}$. Nato proprio per facilitare il riuso di risorse didattiche, SCORM in pratica ha contribuito ad una visione dell'educazione digitale come assemblaggio di parti già pronte, autonome, chiuse. Un oggetto SCORM viene eseguito in una finestra separata, e non è possibile riusarne parti o collegare elementi al suo interno. Adattare una risorsa, trasformarla per raggiungere uno scopo diverso - come propone il documento UNESCO - richiede invece l'intervento a livello di asset interni (estrarre, modificare, cancellare, sostituire) e di relazioni tra gli asset.

Questa possibilità, però, non è legata solo alla gratuità della risorsa: occorre che sia "aperta" in almeno altri due sensi. Il primo è esplicitamente citato: open licensing. Una risorsa, per essere adattabile ad un contesto diverso, deve essere rilasciata dall'autore originario con una licenza che ne permette almeno l'uso e la modifica. Abbiamo visto che questi due diritti fanno parte delle quattro libertà del software libero (in particolare, la 0 e la 1). Un altro modo di descrivere questa apertura è fare riferimento alle licenze Creative Commons, che però funzionano al contrario dei permessi, cioè descrivono in maniera atomica quello che non è possibile fare con un documento. Naturalmente il discorso non si dovrebbe fermare qui: una volta modificata la risorsa,

${ }^{7}$ https://adlnet.gov/projects/scorm/ è possibile ridistribuirla? Con quale licenza? Ovvero: la licenza apposta alla risorsa originaria deve limitare la possibilità di riuso ai soli primi utenti, o deve propagarsi a tutte le opere derivate? Sembra una questione di lana caprina, ma non lo è. Se un docente ha diritto con i suoi studenti di scaricare (gratuitamente o meno) una risorsa, di modificarla e ridistribuirla, la stessa cosa può fare un'impresa attiva nel campo della creazione di contenuti per l'e-learning. In quel caso la risorsa riadattata, migliorata, standardizzata e dotata di qualità professionale, può essere rivenduta? È vero che la nuova risorsa ingloba parte di quella originaria, ma incorpora anche nuovo lavoro e competenze aggiuntive. Come incentivare queste operazioni?

Ma c'è un secondo significato di "aperto" che diventa importante: la risorsa deve essere strutturata in un formato standard e conosciuto, in modo che sia tecnicamente possibile comprenderne la struttura interna. Ad esempio, un sito web realizzato tramite pagine HTML è una risorsa strutturata secondo uno standard pubblico ed è facilmente modificabile. Un documento in PDF che contiene la scansione di un testo (quindi un'immagine) lo è molto di meno. Probabilmente aiuta il riferimento alla classificazione degli Open Data creata da Tim Berners Lee. La risorsa dovrebbe essere disponibile con una licenza aperta ( 1 stella), strutturata ( 2 stelle) e in un formato aperto $(3 \text { stelle })^{8}$.

Abbiamo visto in precedenza come la caratterizzazione delle piattaforme digitali educative come free/open derivi da un loro apparentamento con gli Open Content. Tuttavia questo appoggio, a nostro parere, non risolve i problemi che abbiamo evidenziato.

Il movimento dell'Open Content (e più in particolare, quello per Open Educational Resource) spinge per la gratuità dell'accesso alle risorse educative, con motivazioni che vanno da quelle universali (contrasto al digital divide e della disparità di opportunità per studenti di Paesi diversi) a quelle interne al mondo universitario (l'aumento della qualità derivato da una maggior competizione tra ricercatori e dall'uscita dal circolo vizioso delle riviste). Anche in questo campo, però, pur nella sostanziale adesione agli obiettivi, non tutti concordano sulla strategia da seguire. Di nuovo si oppone una visione della cultura come bene universale astratto, che deve essere disponibile per tutti allo stesso modo, e una visione della cultura come prodotto umano concreto, che ha bisogno di lavoro non solo per la produzione, ma per la valutazione, l'aggiornamento, la distribuzione. Nella prima visione non c'è spazio per le imprese di settore: ma questo non fa altro che invitare soggetti ancora più 
grandi, non specializzati e senza altri interessi che quelli economici, a presentarsi come alfieri di questa offerta "aperta" solo perché gratuita.

Andiamo a leggere la definizione dell'UNESCO che è la seguente: "Open Educational Resources (OER) are teaching, learning and research materials in any medium - digital or otherwise - that reside in the public domain or have been released under an open license that permits no-cost access, use, adaptation and redistribution by others with no or limited restrictions"

Di nuovo c'è una mescolanza di piani: quello dei diritti (licensing) e quello della gratuità (access). Ma mentre nel caso del software, come abbiamo visto, accesso significa leggibilità del codice sorgente (che è la versione del programma che leggerà solo il programmatore), nel caso delle OER accesso significa fruizione della versione rivolta all'utente finale. Accesso libero alle risorse educative significa che né lo studente, come utilizzatore finale, né la scuola o l'università, come riutilizzatori intermedi, devono pagare per ottenere il diritto di consultare la risorsa.

Subito dopo si collegano le OERs agli altri ambiti di conoscenza aperti:

“OER form part of 'Open Solutions', alongside Free and Open Source software (FOSS), Open Access (OA), Open Data (OD) and crowdsourcing platforms."

È la stessa visione del documento su cui ci siamo concentrati all'inizio. Qui, purtroppo, la confusione aumenta. Cosa sono le "crowdsourcing platforms", e perché sono citate tra le Open Solutions? Wikipedia ne è un esempio: progetti portati avanti tramite il lavoro di migliaia di persone che nemmeno si conoscono, ma condividono un obiettivo, un protocollo e un software. È presente, evidentemente, un'apertura (1) che si traduce nel fatto che Wikipedia non fa pagare i propri servizi, e un'apertura (2) che si traduce in un modello democratico di discussione sul contenuto delle pagine che permette a chiunque di correggere e integrare. Ma tra gli esempi di crowdsourcing generalmente riconosciuti c'è anche TripAdvisor. In casi come questo, l'apertura si riferisce solo al metodo di affermazione dell'opinione di un utente su quella di un altro, ma si applica molto poco alla gestione del sistema nel suo complesso: la multa di cinquecentomila euro comminata nel 2014 dall'Autorità Garante della Concorrenza e del Mercato a TripAdvisor (poi annullata dal TAR) era basata sul fatto che la società pubblicizzava il proprio servizio come attendibile perché basato su recensioni autentiche, mentre è noto che le recensioni sono tutte verificate dallo staff interno, che esistono fake

${ }^{9} \mathrm{https} / / / \mathrm{en} . u n e s c o . o r g /$ themes/building-knowledge-societies/oer account e che è possibile farsi scrivere recensioni a pagamento. Anche Wikipedia non è solo il risultato del lavoro volontario di tante persone, ma è anche una vera impresa gestita da una fondazione (Wikimedia) che ha quattrocentocinquanta dipendenti e riceve ogni anno oltre un centinaio di milioni di dollari in donazioni. Parlare di crowdsourcing come modello di condivisione di conoscenza è, da questo punto di vista, piuttosto ingenuo.

Il crowdsourcing è un processo volontario di collaborazione alla realizzazione di un progetto o alla soluzione di un problema; ma non è così lontano da fenomeni come l'utilizzo dei dati di navigazione degli utenti nel web, o nel mondo fisico, per alimentare e addestrare modelli di machine learning. Anche in questi casi c'è una collaborazione di migliaia di utenti, e c'è una fruizione gratuita di un servizio. Ma c'è anche un evidente vantaggio per chi gestisce il servizio, o tramite la vendita di spazi pubblicitari, o tramite la raccolta di dati. Qui è davvero difficile riconoscere l'aspetto "aperto", e a ben guardare anche la gratuità è relativa: si tratta di uno scambio tra servizi e dati.

Non vedere gli aspetti economici e gli interessi di chi fornisce queste piattaforme per un uso apparentemente "libero" rende il discorso troppo astratto. Non distinguere tra imprese all-market e imprese specializzate, tra imprese che sviluppano secondo il modello opensource e imprese che forniscono servizi gratuiti è un'imprecisione che può cambiare molto del modo in cui i servizi educativi digitali saranno fruiti e gestiti nel prossimo futuro.

Anche in questo caso, come per il software, richiedere che le risorse siano "open" nel senso di "ad accesso libero" significa scaricare il costo del loro progetto, realizzazione e manutenzione su qualcun altro. Anche una volta pubblicate, le OER devono essere verificate e aggiornate continuamente. Chi può sostenere questi costi? (Downes 2007; Sclater 2010). Tradizionalmente si tratta di fondazioni (come la Hewlett Foundation), grandi Università o reti di Università, oppure enti pubblici (a livello nazionale o sovranazionale) che finanziano progetti. In pratica le OER sono svincolate dal mercato, ma non per questo hanno un costo globale nullo se si vuole che siano anche di qualità. È accaduto in passato che interi repository di risorse siano stati sviluppati con fondi pubblici e poi abbandonati a causa dell'esaurirsi del progetto che quelle risorse aveva prodotto. Questo modello, ancora una volta, rischia di escludere i produttori di contenuti educativi, soprattutto i più piccoli, privilegiando quelli che hanno la possibilità di regalare porzioni di contenuti un po' obsoleti in attesa di far pagare le versioni aggiornate, secondo il modello freemium. 


\section{UN ECOSISTEMA SOSTENIBILE}

L'approccio implicito nel documento della Commissione UNESCO è, a nostro parere, limitato e limitante, perché non tiene in considerazione l'esistenza o almeno la possibilità di imprese private che lavorano dentro lo spazio pedagogico, e che producono software libero, rilasciandolo con licenze aperte. Escludendo queste imprese private (e quindi il profitto che le mantiene) dal settore, si finisce per costruire soluzioni insostenibili, perché basate su finanziamenti pubblici temporalmente e quantitativamente limitati; in sostanza si finisce per lasciare il campo libero alle grandissime imprese del digitale generico, che possono permettersi di non ricavare profitto dai servizi per il mondo educativo perché hanno altrove i loro veri interessi.

Dovrebbe essere invece immaginata una collaborazione trasversale tra pubblico e privato, profit e non profit, basato sul riconoscimento dei legittimi interessi, che devono essere trasparenti e regolati.

Questa collaborazione dovrebbe partire dalla raccolta dei bisogni di tutti i portatori di interessi (studenti, docenti, amministrativi, dirigenti; ma anche i bisogni dei lavoratori del comparto: programmatori, progettisti di corsi, editori e sviluppatori di contenuti).

In secondo luogo, dovrebbe essere costituito - in maniera leggera, aperta, anche remota - un comitato che veda al suo interno rappresentanti non solo del mondo della ricerca universitaria (nelle due aree, informatica e pedagogia) ma di tutti i portatori di interesse, ovvero docenti, studenti e persino i produttori delle piattaforme, che devono poter esprimere il loro punto di vista.

Questo comitato dovrebbe lavorare al design di soluzioni, che non sono necessariamente "la" piattaforma nazionale, ma piuttosto un ecosistema sostenibile di piattaforme diverse basato su questi tre punti:

- Linee guida di livello nazionale che dicano cosa deve essere e cosa deve fare una piattaforma digitale per la didattica (licenze, supporto, privacy, funzioni, accessibilità). Linee guida estese, dettagliate, pubbliche che dovranno essere riviste ogni anno.

- Registro nazionale dove venga iscritta ogni piattaforma che sia conforme alle linee guida di cui sopra e che sia in uso dalle scuole. Il registro servirà a rendere possibile la verifica per ogni piattaforma di questa conformità, ma anche a permettere un'interrogazione automatica, una scoperta dei servizi messi a disposizione da quella piattaforma verso l'esterno: assets semplici, attività, interi corsi.

- Protocolli di interscambio di metadati e di contenuti tra piattaforme. Per "metadati" si intendono gli indici dei corsi e delle attività (con le licenze relative), con le proprietà che li descrivono, secondo una classificazione univoca e pubblica. Per contenuti intendiamo non solo i singoli asset, ma interi blocchi, sequenza, mappe, fino ad attività intere.

In questo modo sarebbe possibile avere, anziché una sola piattaforma di stato, tante piattaforme diverse, adatte a contesti diversi, realizzate e gestite da enti pubblici o privati, purché siano conformi agli standard definiti e condivisi.

Naturalmente anche i protocolli di scambio dovrebbero essere aperti. Questo significa che la possibilità di scambiare informazioni tra le piattaforme dovrebbe essere regolata in termini non solo dei formati in cui i dati vengono rappresentati (ad esempio JSON o XML) o di protocolli generali di comunicazione (ad esempio REST o SOAP), ma ad un livello più dettagliato, specifico del contesto educativo. Le due iniziative a cui guardare sono probabilmente LTI $^{10}$ di IMSGlobal, che permette di integrare contenuti e attività esterni all'interno di una piattaforma, e $\mathrm{xAPI}^{11}$ di ADL/Rustici, che permette di inviare e registrare eventi e attività compiute ad un Learning Record Store che agisce come un archivio imparziale.

Esistono però anche dei protocolli molto più generali per la definizione di webservices (come WSDL ${ }^{12}$ ) che si potrebbero usare come base per definire un protocollo a cui tutte le piattaforme dovrebbe conformarsi, e che stabilisca come ottenere l'elenco dei servizi, l'elenco dei contenuti (filtrati per area o per età del destinatario), le condizioni per il riuso, la data di aggiornamento, gli autori, etc.

In conclusione, ci auguriamo fortemente che le idee espresse dalla Commissione UNESCO divengano patrimonio comune, ma anche che si esca dalla visione dicotomica pubblico/privato che emerge in alcuni punti chiave di quel documento, e si recuperi il significato originale di "open" in termini di libertà e non di gratuità. Come risultato, includendo le imprese (per lo meno quelle specializzate e disposte a lavorare in un'ottica open), si avrebbe una maggiore varietà di proposte; e la varietà è sempre preferibile al monopolio, perché nel suo complesso è più robusta e sicura. Aprire la collaborazione al progetto di un ecosistema di piattaforme spingerebbe anche la nascita di nuove proposte imprenditoriali etiche, cioè non orientate al solo business, e ad orizzonte controllato, cioè che non abbiano come obiettivo quello di conquistare l'intero mercato estromettendo tutti gli altri.

Vedremmo allora nascere una varietà di piattaforme e applicazioni più semplici, dedicate a diversi livelli di

\footnotetext{
${ }^{10} \mathrm{https} / /$ www.imsglobal.org/spec/lti/v1p3/

${ }^{11}$ https://adlnet.gov/projects/xapi/

${ }^{12} \mathrm{https} / / /$ en.wikipedia.org/wiki/Web_Services_Description_Language
} 
scuola, in grado di colloquiare tra loro per far emergere strutture di livello superiore. Piattaforme che con la loro produzione, manutenzione, arricchimento, non si limiterebbero a fornire servizi educativi digitali nel pubblico, ma permetterebbero la nascita di un tessuto di micro- e piccole imprese locali specializzate.

\section{BIBLIOGRAFIA}

Downes, S. (2007). Models for Sustainable Open Educational Resources. Interdisciplinary Journal of Knowledge and Learning Objects, 3(1), 29-44.

Free Software Foundation (2021). Cosè il software libero. Traduzione italiana di Dino Distefano, Francesco Potortì, Alessandro Rubini, Paola Blason, Giorgio V. Felchero, Andrea Pescetti, Marco Calegaro. https:// www.gnu.org/philosophy/free-sw.html

International Commission on the Futures of Education (2020). Education in a post-COVID world: nine ideas for public. UNESCO. https://unesdoc.unesco.org/ ark:/48223/pf0000373717/

Ministero dell'Istruzione, dell'Università e della Ricerca (2020). Coronavirus, pubblicate due call per sostenere la didattica a distanza. https://www.miur.gov.it/ web/guest/-/coronavirus-pubblicate-due-call-per-sostenere-la-didattica-a-distanza

Morozov, E. (2013). Open and Closed. New York Times, retrieved from https://www.nytimes. com/2013/03/17/opinion/sunday/morozov-open-andclosed.html

Sclater, N. (2010). Open Educational Resources: Motivations, Logistics and Sustainability. In Ferrer, N. F. \& Alonso, J. M. (Eds) Content Management for E-Learning. Springer. Retrieved from http://sclater.com/ papers/OER-Motivations-Logistics-and-Sustainability-Sclater.pdf 